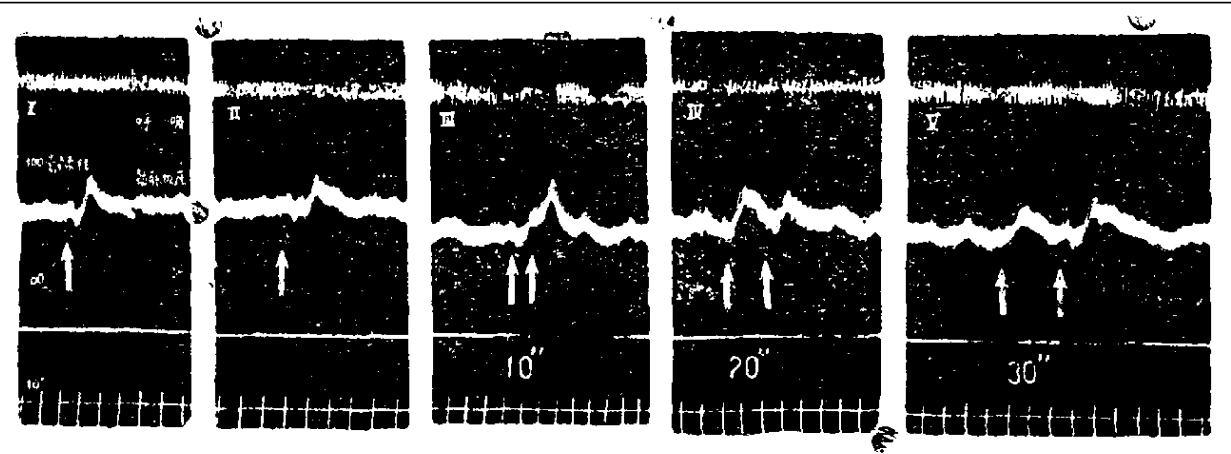

图 2 先后刺激雨段小陊的化学感受器的时成关系

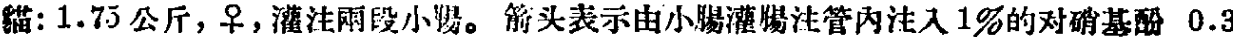

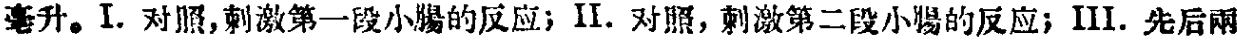
次刺激相隔 10 秒, 刺激两段小晹时所得的綜合反应; IV. 先后丽次刺激相隔 20 秒刺激 两段小㨉，第二次刺激第二段小腸的反应比对照小; V. 先后兩次相隔 30 秒刺激雨段小

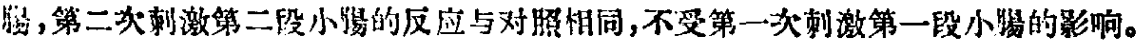

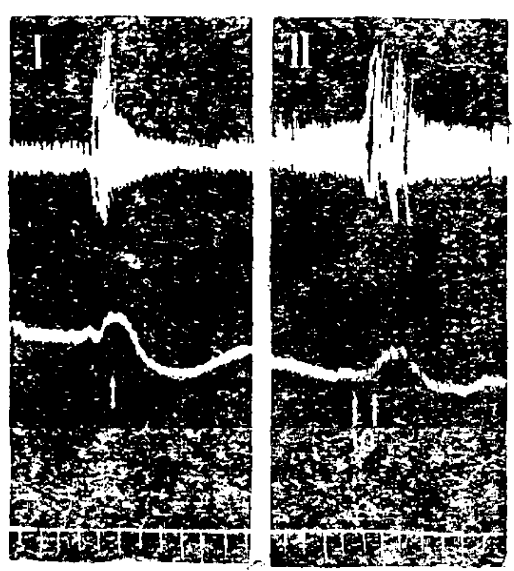

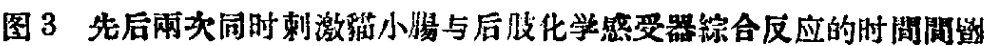

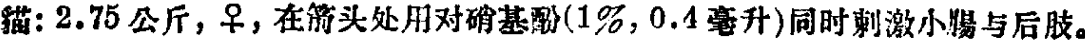

这很可能是一种䇋导的关系。

沈泰春 刘世樟 徐树篻

\section{水稻土中决定嵫化还原电 位的体系問題}

关于土壤中决定氧化还原电 位的体系問題, 在土壇化学上有 着不同的意見 ${ }^{[1]}[2][8][4]$ 。我們 在太湖地区中性沉积水稻士（白 土）中測定氧化还原狀况的季节 变化时, 此較了代撸性 (包拈水 溶性）铁的会量与载化还原电 位的关系(如图)。

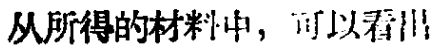
几个主要趋势。总的說，电位润 数值急低，則低价鉄鲜的含量忿 高; 但是兩种元薪与氧化还原电 位的关系, 表現了不同的悱沙。 一般在电位高于約 350 篦伏时， 低价鉄的数量就很少，但是作
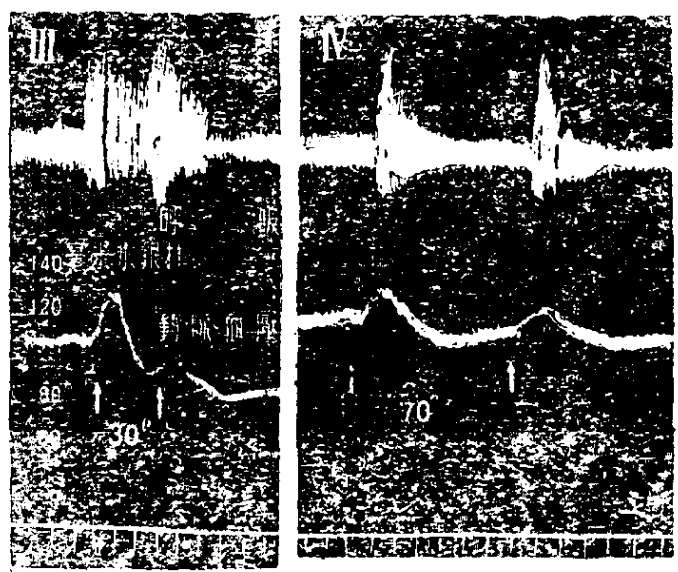

500 瓷伏以上时，仍可有相

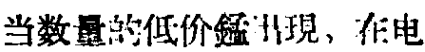

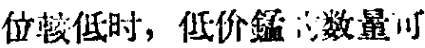

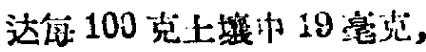
相当全部的 $(0.026 \%)$ 中的 $73 \%$ ，亦垖絶大多数的悇都 变成了还原斨态。这涗叮捁 的化合物轻鉄化合物更易于 被还原。男一方面也說明在 由还原条件較强的狀況变为 氧化条件較强的颀汾时，低 价铁較领更易于被氛化。铁 和的这种差别，特合于二 种元素的电化学性質。

但值得注意的是，在电 位很低时，低价铁的数量边 可以扑不等样多，例如任烤 町后重新灌水后三天，电位 降至 87 毫伏时，低价鉄仅为 往. 100 克土壤中18.7畧克; 反之, 在电位很高时, 也订 能有大吾低价鉄存存: 例如 由于烤田的結果，电位升至 517 毫伏时，而上堆仍含有 住: 100 克土中 85.7 管克低 价鉄。这些情洗說明了一个 平要的閒題: 低价铁离了扑不是决定电位的主要体 系。当然，由于士埣中的含鉄量很高，在低价鉄达栵 住 100 克比㳟中 300 毫克时，也不过是全部鉄中 的 5 $-10 \%$ 成为还原狀涊，所以作为一个等化还原体系来 看，鉄还是很守要的。可以說，鉄化合物在这方面的

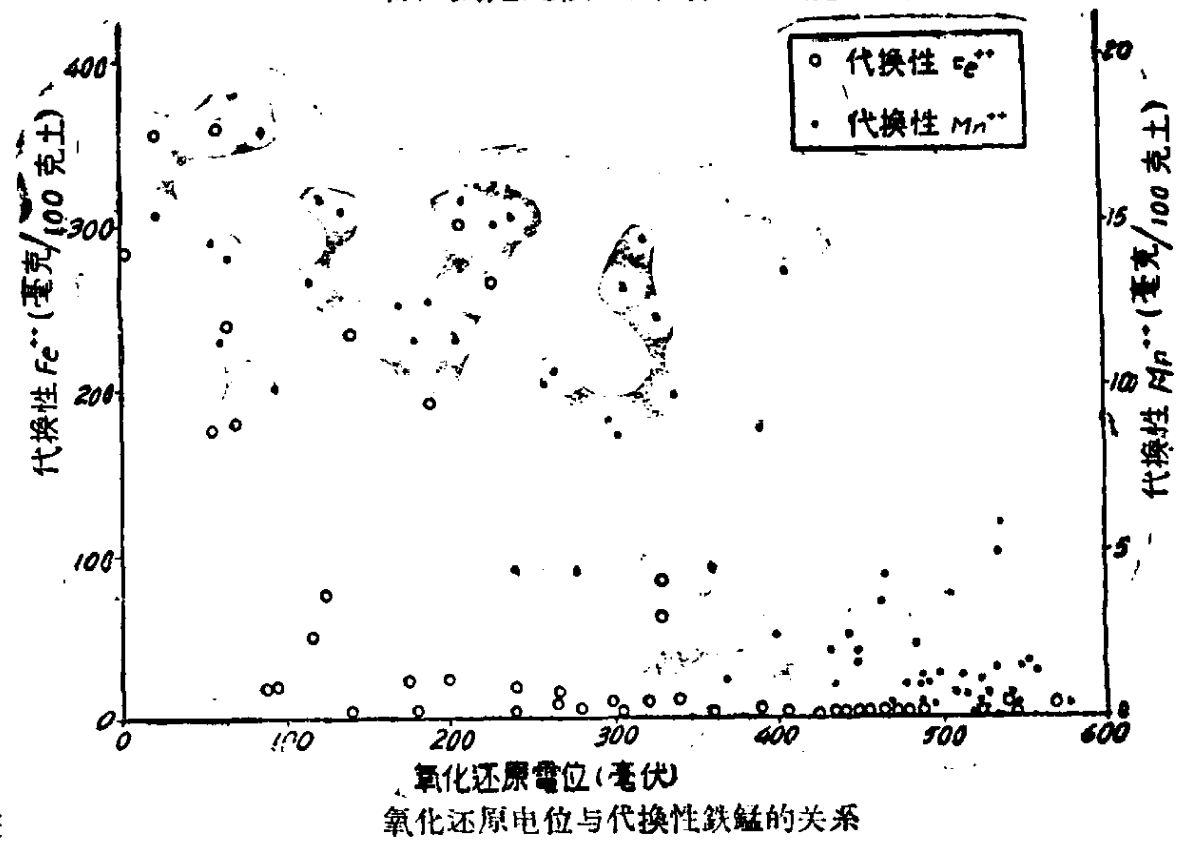


作洲，与酸硆㗔衡液中米解离的部分相似。

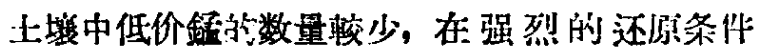

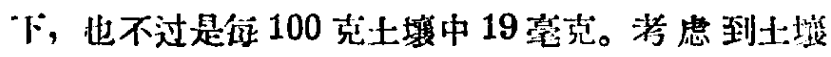

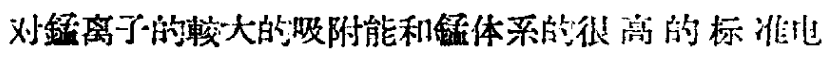
位，所以也不能認为銛离子是决定电位的主要体系。

根据田間测是結果, 在六月中旬, 田面水中的电

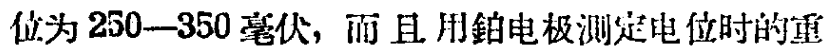
現性很羔, 說朋此时水中除氧以外，其他能起本衡作 HJ於体系的数量很小。可以認为, 在电仿高于約 300 综伏时, 在上壤中的一般 $\mathrm{pH}$ 条作下，时于已超过一 般已知有机体系的标准电位数值很多, 所以此时溶解 的气在决定电位方面, 逐漸起有較大的作川。但是

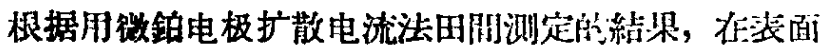
瀵水时, 土㙍棑层中溶解的氧气的数量极微, 所以应

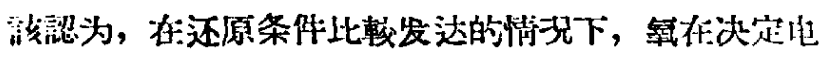
位方面所起的作用很小; 此时决坚电位的, 应該主要 是有机体系。

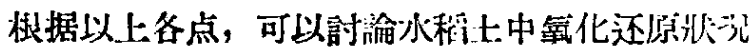

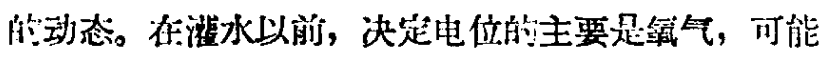
还有极少量的有机体系，尤其是在通气性较参，中任。

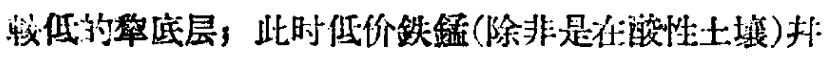
不H現。在灌水以后, 由于微生物的活动, 使氧气的 数量濑少, 区此等在决定电位方面所起的作州逐旉减 少，而有机体系的作用潮大。这时时于鉃鎾体系的化

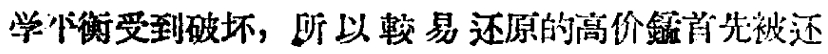
原，然后是大量的鉄被还原。这时如朵电位隆低的这 度很快, 在一定时期內，鉄銛特别是鉄的变化速度， 可以迫随不上电位(此时主要目有机体系所决管)的变 化速度。何如存湤水 5 天后 (6 月 19日), 表上的扎

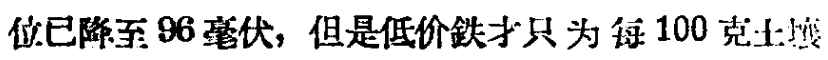
中 20 莚克， 10 毫克，到 6 月 28 日，低价鉄与 朴至 74 笔克, 船升至 16 撉克。但郎使是在与有机体 系大致达到动态本衡的情况下，低价鉄銛在决定电储。 方面所起的作州，也只佔一本底存 ${ }^{[0]}$, 而还有一作 作用是易于有机体系。在烤田或落水时, 氧气治进 来, 使电位升高, 㚘且使低价鉄銛, 肖先是鉄逐鿰被 化; 这时如果土壤变干的速度很快, 则任一管时时 队, 鉄的特别是的变化速度, 可以道随不上毛位。 (此时主要的氛所次定)的变化速度，例如在 7 月 26 II，耕作层的水分已目原来的 $45 \%$ 降当 $27 \%$ ，电值。

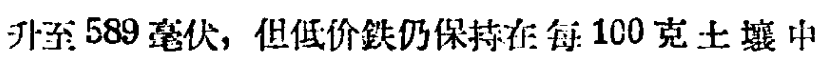

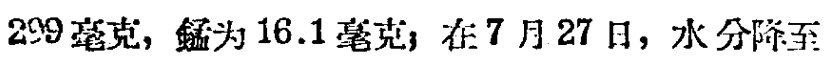
$24 \%$ ，电位升至 610 毫伏，低价鉄仍保持在 9.8 毫克,

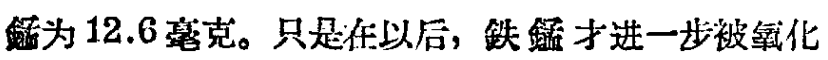
而消头。

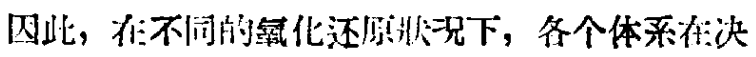

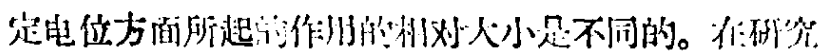

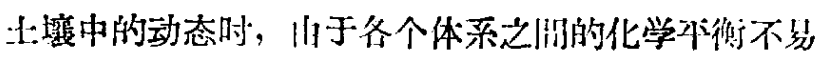
建立, 这种关柔就更复杂一些。不能認为在一边㤔况

下，电仿都是的闪一种体系沂决定。

\section{于天仁謝建昌 㛫国治

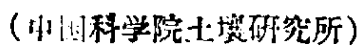

[1] Pearsall, W. H. and Mortimer, C. H., 193.), J. Ecology, 27, 483-501.

[2] Bradfield, R. et al, 1934, Cornell Un:v. Agric. Exp. Sta. Bul., 592 .

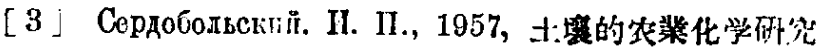
法, 152-207 (科学蜘社)。

[4] Ponuamperuma, F. N., 1955, Thesis Corncll Univ. (Minofilm).

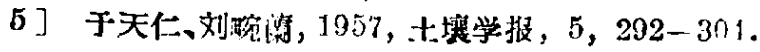

\section{集宁石(Jiningite) 一种新的針石变种}

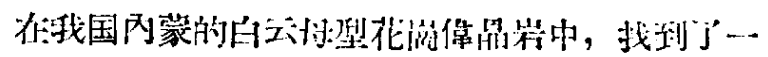

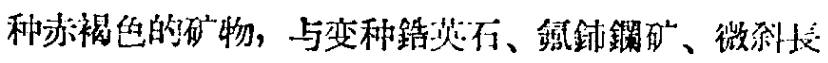
不、日云妇、石英及柘榴石等矿物基生。此盕 $=4.0108$,

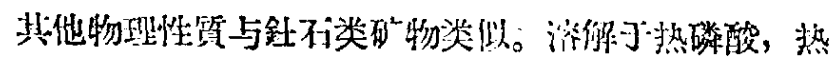

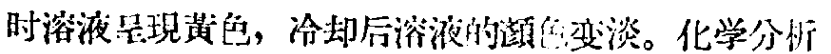
的紸桃如下:

\begin{tabular}{|c|c|}
\hline 成 等 & 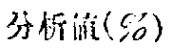 \\
\hline $\mathrm{CaO}$ & 7.43 \\
\hline $\mathrm{MgO}$ & 0.49 \\
\hline $\mathrm{MnO}$ & thes \\
\hline $\mathrm{PbO}$ & 0.52 \\
\hline $\mathrm{Fe}_{2} ?_{3}$ & 17.28 \\
\hline $\mathrm{Al}_{2} \mathrm{O}_{3}$ & 1.48 \\
\hline $\mathrm{TR}_{2} \mathrm{O}_{3}$ & 1.84 \\
\hline $\mathrm{Th}_{1} \mathrm{O}_{2}$ & 44.83 \\
\hline $\mathrm{ZrO}_{3}$ & 0.95 \\
\hline $\mathrm{U}_{3} \mathrm{O}_{8}$ & 0.16 \\
\hline $\mathrm{SiO}_{2}$ & 7.13 \\
\hline $\mathrm{P}_{2} \mathrm{O}_{5}$ & i. os \\
\hline $\mathrm{V}_{2} \mathrm{O}_{5}$ & 0.07 \\
\hline $\left.\mathrm{H}_{2} \mathrm{O}^{+}\right)$ & 7.05 \\
\hline $\mathrm{H}_{2} \mathrm{O}^{\prime}$ & 2.84 \\
\hline
\end{tabular}

在化学組成上, 与一般的鐩不淡矿物比较, 木研 物有以下几个瑔点:

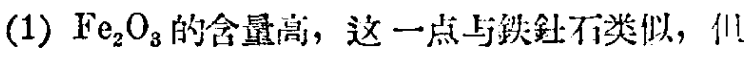

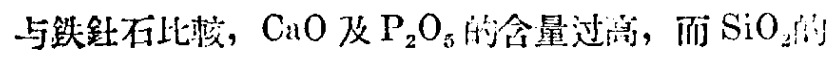

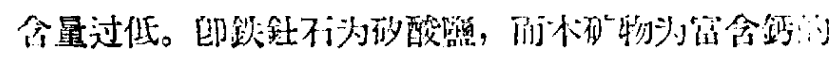
磁故酸㜻。

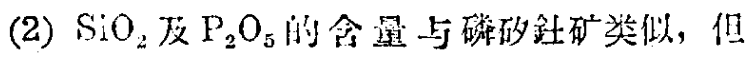

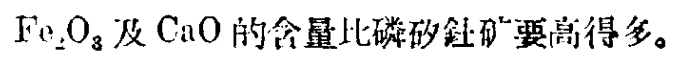

